\title{
Expression of immune checkpoints and T cell exhaustion markers in early and advanced stages of colorectal cancer
}

\author{
Reem Saleh ${ }^{1} \cdot$ Rowaida Z. Taha $^{1} \cdot$ Salman M. Toor ${ }^{1} \cdot$ Varun Sasidharan Nair $^{1} \cdot$ Khaled Murshed $^{2} \cdot$ Mahwish Khawar $^{3}$. \\ Mahmood Al-Dhaheri ${ }^{3} \cdot$ Mahir Abdulla Petkar ${ }^{2} \cdot$ Mohamed Abu Nada $^{3} \cdot$ Eyad Elkord $^{1}$ (1)
}

Received: 16 February 2020 / Accepted: 27 April 2020 / Published online: 11 May 2020

(c) The Author(s) 2020

\begin{abstract}
Despite recent advances in colorectal cancer (CRC) treatment, a large proportion of patients show limited responses to therapies, especially in advanced stages. There is an urgent need to identify prognostic biomarkers and/or therapeutic targets in advanced stages, aiming to improve the efficacy of current treatments. We aimed to determine prognostic biomarkers in tumor tissue and circulation of CRC patients, with a special focus on $\mathrm{T}$ cell exhaustion markers. We found that mRNA levels of PD-1, TIM-3, CTLA-4, TIGIT, CD160, CD244, KLRG1, TOX2, TOX3, Ki-67, and PRDM1 were elevated in CRC tumor tissues. We also investigated differences in gene expression between early and advanced disease stages. We found that TOX and potentially TIM-3, CTLA-4, VISTA, TIGIT, KLRG1, TOX2, SIRT1, Ki-67, and Helios mRNA levels in tumor tissue were elevated in advanced disease stages, suggesting their potential roles in CRC progression. In contrast, PD-1 and CD160 levels in tumor tissue were downregulated in advanced stages. In the circulation of CRC patients, mRNA levels of PD-1, VISTA and LAG-3 were higher than those of healthy individuals. Moreover, in circulation, PD-1, CTLA-4 and TIGIT mRNA levels were reduced in advanced stages. Interestingly, levels of PD-1 in both tumor tissue and circulation were reduced in advanced stages, suggesting that targeting PD-1 in patients with advanced stages could be less effective. Altogether, these findings suggest some potential $\mathrm{T}$ cell exhaustion markers that could be utilized as prognostic biomarkers and/or therapeutic targets for CRC. However, further investigations and validations in larger cohorts are required to confirm these findings.
\end{abstract}

Keywords Colorectal cancer · Immune checkpoints · T cell exhaustion · Prognostic biomarker · Therapeutic target

$\begin{array}{ll}\text { Abbreviations } \\ \text { Blimp1 } & \text { B lymphocyte-induced maturation protein-1 } \\ \text { CRC } & \text { Colorectal cancer } \\ \text { CTLA-4 } & \text { Cytotoxic T-lymphocyte-associated protein } 4 \\ \text { KLRG1 } & \begin{array}{l}\text { Killer cell lectin-like receptor subfamily G } \\ \text { member 1 }\end{array} \\ \text { LAG-3 } & \text { Lymphocyte-activation gene } 3\end{array}$

Electronic supplementary material The online version of this article (https://doi.org/10.1007/s00262-020-02593-w) contains supplementary material, which is available to authorized users.

Eyad Elkord

eelkord@hbku.edu.qa; e.elkord@salford.ac.uk

1 Cancer Research Center, Qatar Biomedical Research Institute (QBRI), Hamad Bin Khalifa University (HBKU), Qatar Foundation (QF), P.O. Box: 34110, Doha, Qatar

2 Department of Pathology, Hamad Medical Corporation, Doha, Qatar

3 Department of Surgery, Hamad Medical Corporation, Doha, Qatar
PBMC Peripheral blood mononuclear cell

PD-1 Programmed cell death-1

PRDM1 PR domain zinc finger protein 1

SIRT1 Silent information regulator 1

TIGIT T cell immunoreceptor with Ig and ITIM domains

TIM-3 T-cell immunoglobulin and mucin domain-3

TME Tumor microenvironment

TOX Thymocyte selection-associated high-mobility group box

Treg T regulatory cell

VISTA V-domain Ig-containing Suppressor of T cell Activation

\section{Introduction}

Cancer immunotherapies, including recombinant cytokines, immune checkpoint inhibitors (ICIs), adoptive T cell therapies, and vaccines, have revolutionized the treatment of 
several solid tumors and hematologic malignancies [1]. Approved ICIs in the form of monoclonal antibodies against cytotoxic T-lymphocyte-associated antigen 4 (CTLA4), programmed cell death protein 1 (PD-1), or its ligand PD-L1 have enhanced clinical responses in patients with advanced melanoma, lung, kidney, bladder cancer; however, limited response rates were evident in many other patients due to primary or acquired resistance $[2,3]$. The sensitivity of host response to ICIs is largely dependent on the tumor type and preexisting anti-tumor immunity [3]. Combining ICIs with other therapeutic agents targeting T cell exhaustion holds the potential to restore more potent anti-tumor immune responses, enhance the clinical efficacy of current treatments and overcome the resistance currently seen in many cancer patients [4].

As cancer cells divide, they acquire genetic and epigenetic alterations giving rise to new cancer clones with different molecular profiles and inheritable traits favoring tumor growth/survival and immune response escape [5]. These alterations can influence the expression of immune checkpoints (ICs), some of which might lead to T cell exhaustion $[6,7]$. T cell exhaustion is a status characterized by the loss of effector function, including proliferation, release of cytokines, and secretion of cytolytic molecules, due to continuous antigen stimulation in pathological contexts, such as chronic inflammatory conditions, viral infections, and cancer [7]. Immunosuppressive cells and cytokines present in the TME can also contribute to this exhausted phenotype [6]. Additionally, T cell exhaustion is induced by the overexpression of multiple inhibitory ICs, along with other T cell exhaustion markers, such as transcription factors $[8,9]$. Inhibitory ICs, such as PD-1, CTLA-4, T cell immunoglobulin and mucin domain-containing protein 3 (TIM-3), lymphocyte-activation gene 3 (LAG-3), V-domain Ig suppressor of T cell activation (VISTA), T cell immunoreceptor with Ig and ITIM domains (TIGIT), CD160, CD244, and killer cell lectin-like receptor subfamily G member 1 (KLRG1), can induce $\mathrm{T}$ cell dysfunction/exhaustion via direct mechanisms through the interactions with their ligands, leading to impaired $\mathrm{T}$ cell activation, inhibition of $\mathrm{T}$ cell proliferation and impaired cytokine release [8, 10-12]. Thymocyte selection-associated high-mobility-group box (TOX) family members and B lymphocyte-induced maturation protein-1 (Blimp1) are transcription factors, which can indirectly lead to immune evasion and impaired $\mathrm{T}$ cell activation by altering cell differentiation and gene expression [9, 13, 14]. Other markers, such as SIRT1, Ki-67 and Helios, can also influence $\mathrm{T}$ cell function and induce immune evasion via indirect mechanisms, and have been implicated in cancer progression [15-18]. However, little is known about the contribution of some of these markers to CRC progression and metastasis, and how their expressions differ during early and advanced stages of the disease.
In this study, we investigated gene expression levels of various inhibitory ICs including PD-1, TIM-3, LAG-3, VISTA, CTLA-4, TIGIT, CD160, CD244, and KLRG1 in the tumor tissue of CRC patients. In addition, expression levels of markers related to $\mathrm{T}$ cell exhaustion, cell survival, senescence, proliferation and differentiation; for example, members of the TOX family, silent information regulator 1 (SIRT1), PRDM1 (gene name for Blimp1), Helios and Ki-67, were also determined in tumor tissues of CRC patients. These genes were selected based on their importance in cancer progression and $\mathrm{T}$ cell exhaustion as implicated by preclinical models and clinical data; however, their expression profiles in CRC and their expression patterns during the onset and progression of disease have not been addressed yet. Gene expression analyses to determine differences between early and advanced disease stages could be a useful tool to provide insights into the transcriptomic changes during disease onset and progression [19, 20]. Overall, our data provide novel insights into some of immune signature genes, which are altered during early and advanced stages of CRC and could be utilized as prognostic biomarkers and/or therapeutic targets for CRC.

\section{Materials and methods}

\section{Sample collection and storage}

PBMCs were isolated from peripheral blood samples of 30 healthy donors (HD) and 68 CRC patients by density gradient centrifugation using Histopaque-1077 (Sigma-Aldrich, Missouri, USA). PBMCs were frozen in cryovials at a density of $5-10 \times 10^{6}$ cells in $1 \mathrm{ml}$ of freezing medium [ $(10 \%$ dimethylsulfoxide (DMSO; Sigma-Aldrich), 50\% fetal calf serum (FCS; Hyclone, GE Healthcare Life Sciences, Utah, USA), and 40\% RPMI-1640 medium (Life Technologies, New York, USA)], and stored in liquid nitrogen to be used in batches in subsequent experiments. Tumor tissues (TT) and paired-adjacent normal tissues (NT) were obtained from 70 CRC patients who underwent surgery at Hamad Medical Corporation, Doha, Qatar. TT and NT sections were identified by microscopic pathological examination by the pathologist. Typically, TT specimens were taken from tumor core, while normal tissue sections were taken away from the tumor margins and as deemed relevant by the pathologist. Tissue specimens were cut into pieces, snap-frozen in liquid nitrogen, and stored at $-80{ }^{\circ} \mathrm{C}$ to be used in subsequent experiments.

All patients included in the study were treatment-naïve prior to surgery and provided written informed consent prior to sample collection. Table 1 shows the details for the healthy donors included in this study and the clinical and pathological characteristics of all participating patients. All 
Table 1 Characteristic features of study populations

\begin{tabular}{llll}
\hline & Healthy donors (HD) & $\begin{array}{l}\text { CRC patients (tissue } \\
\text { samples) }\end{array}$ & $\begin{array}{l}\text { CRC patients } \\
\text { (PBMC sam- } \\
\text { ples) }\end{array}$ \\
\hline Number & 30 & 70 & 68 \\
Age (median) & $30(20-47)^{\S}$ & $59(23-96)^{\S}$ & $60(23-96)^{\S}$ \\
Gender (Male: Female) & $14: 16$ & $47: 23$ & $42: 26$ \\
TNM stage & & & \\
I & & 5 & 10 \\
II & & 24 & 23 \\
III & 31 & 24 \\
IV & 10 & 11 \\
Tumor budding & & 29 \\
Low & 31 & 19 \\
Intermediate & 20 & 20 \\
High & 19 & \\
\hline
\end{tabular}

$C R C$ colorectal cancer

${ }^{\S}$ Median range experiments were performed in accordance with relevant guidelines and regulations. This study was executed under ethical approvals from Hamad Medical Corporation, Doha, Qatar (protocol no. MRC-02-18-012) and Qatar Biomedical Research Institute, Doha, Qatar (Protocol No. 2017-006 and 2018-018).

\section{RNA extraction and reverse transcription}

Total RNA was extracted from PBMC of HDs and CRC patients, and NT/TT of CRC patients, using RNA/DNA/ Protein Purification Plus Kit (Norgen, Ontario, Canada). Briefly, PBMCs were washed with $1 \times$ phosphate-buffered saline $(1 \times \mathrm{PBS})$ and resuspended with lysis buffer. TTs and NTs were also resuspended with lysis buffer, but homogenized using a tissue homogenization set (Bioneer Inc., Daejeon, South Korea). RNA extraction was performed following the manufacturer's protocol. RNA concentrations were measured using NanoDrop 2000 (Thermo scientific). $1 \mu \mathrm{g}$ of RNA was reverse-transcribed into cDNA using QuantiTect Reverse Transcription Kit (Qiagen, Hilden, Germany).

\section{Quantitative real-time reverse transcriptase PCR (qRT-PCR)}

qRT-PCR was performed using QuantStudio 6/7 Flex realtime PCR system (Applied Biosystems, California, USA), for the genes listed in Supplementary Table 1, with PowerUp SYBR Green Master Mix (Applied Biosystems). Quantification of relative gene expression was determined using $2^{-\Delta \Delta C T}$, and normalized to $\beta$-actin. Sequences for the primers are shown in Supplementary Table 1.
For circulation, the $C_{\mathrm{t}}$ values of target gene from the HDs were initially normalized to $\beta$-actin. The relative expression of target gene from each patient was calculated by normalizing the expression of each sample with average expression of the same gene from HDs, and subsequently $2^{-\Delta \Delta C T}$ method was applied. Additionally, for CRC tissues, the relative expression was calculated by normalizing the expression of each TT sample to its corresponding NT after the initial normalization to $\beta$-actin. Therefore, the relative fold change of each TT sample was calculated by keeping the value of corresponding NT sample as constant " 1 ". The statistical significance was calculated by performing unpaired and paired $t$ tests for circulation and CRC tissues, respectively $[21,22]$.

\section{Statistical analyses}

Statistical analyses were performed using GraphPad Prism 8 software (GraphPad Software, California, USA). Paired and unpaired $t$ tests were performed on samples that passed the Shapiro-Wilk normality test. Non-parametric Wilcoxon signed-rank tests or Mann-Whitney $U$ tests were performed for samples that did not show normal distribution. Mann-Whitney $U$ test was used to compare the differences between early (stages I and II) and advanced (stages III and IV) stages. Comparisons of gene expression among different tumor budding grades were performed using Kruskal-Wallis test one-way analysis of variance (ANOVA). A $P$ value of $>0.05$ was considered statistically nonsignificant. The $P$ values are represented as follows; $* * * P<0.001, * * P<0.01$ ,${ }^{*} P \leq 0.05$. Data are presented as mean \pm standard error of the mean (SEM). 


\section{Results}

\section{Elevated expression of immune checkpoints and T cell exhaustion-related genes in the tumor tissue of CRC patients}

Increased expression of multiple immune checkpoints and $\mathrm{T}$ cell exhaustion markers in the tumor tissue of cancer patients has been associated with the suppression of anti-tumor immunity and resistance to therapy [3, 23]. Therefore, we investigated the expression levels of different genes that have been previously implicated in immune evasion and $\mathrm{T}$ cell exhaustion in the tumor tissue (TT) and paired-normal tissue (NT) of CRC patients (Fig. 1). The panel of genes consisted of well-known inhibitory ICs, PD-1, TIM-3, CTLA-4, VISTA, LAG-3, TIGIT, CD244, CD160, and KLRG1 [24], in addition to T cell exhaustion-related markers namely TOX, TOX2, TOX3, TOX4 [9, 14], Helios, PRDM1 (Blimp1), Ki-67 [25], and other markers involved in tumorigenesis such as SIRT1 [15].

We found that mRNA levels of PD-1, TIM-3, CTLA4, TIGIT, CD160, CD244, and KLRG1 were significantly higher in TT, compared to NT (Fig. 1a). LAG-3 and VISTA mRNA levels were relatively similar in TT and NT (Fig. 1a). We also investigated the expression of TOX family members and other tumorigenic markers. We found that TOX mRNA level was relatively similar in TT and NT, while TOX2 and TOX3 mRNA levels were significantly higher in TT (Fig. 1b). TOX4 mRNA level was upregulated in TT compared with NT; however, it did not reach statistical significance (Fig. 1b). SIRT1, Ki-67, and PRDM1 mRNA levels were also upregulated in TT (Fig. 1c); however, mRNA levels of Helios in TT and NT were relatively similar (Fig. 1c).

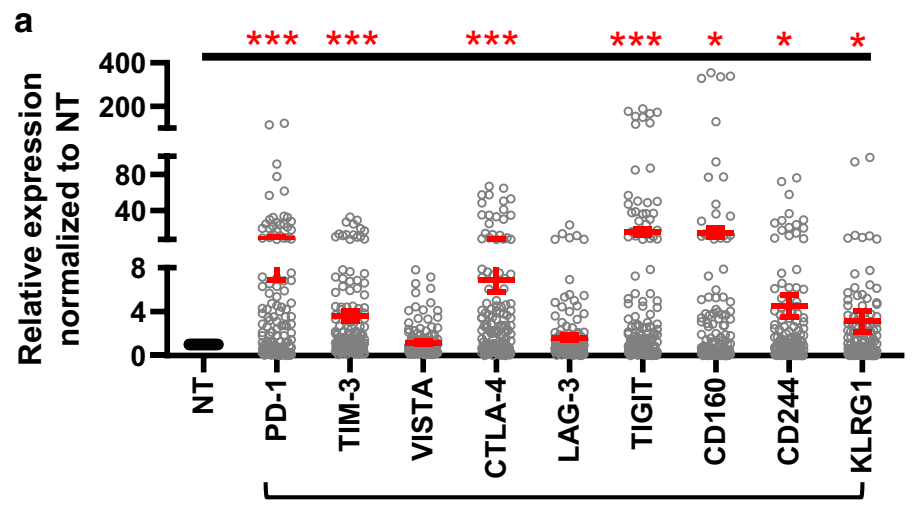

TT

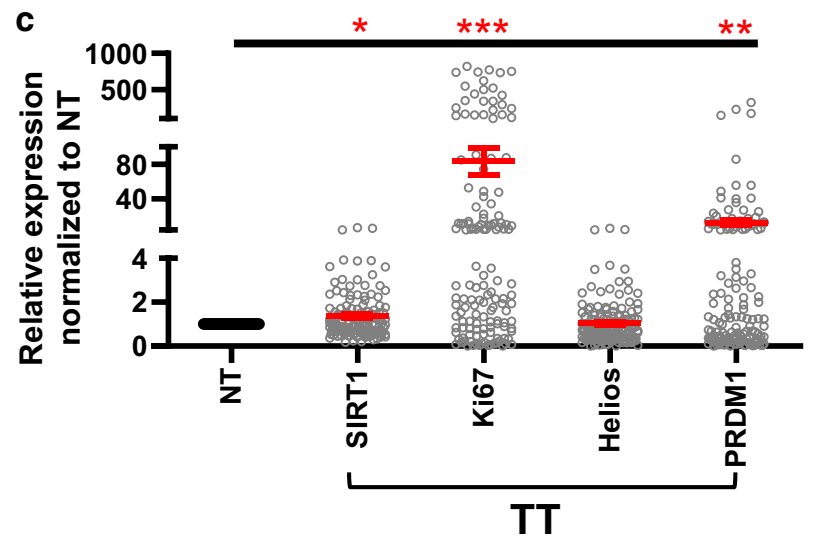

Fig. 1 Gene expression of immune checkpoints, T cell exhaustionrelated markers and cancer progression-related markers in colorectal cancer tissues. RNA was isolated from normal and tumor tissues of 70 patients, and reverse transcribed to cDNA. Quantitative RT-PCR was performed to assess the expression levels of immune checkpoints (PD-1, CTLA-4, TIM-3, VISTA, CTLA-4, LAG-3, TIGIT, CD160, CD244, and KLRG1) (a), TOX family members (TOX,

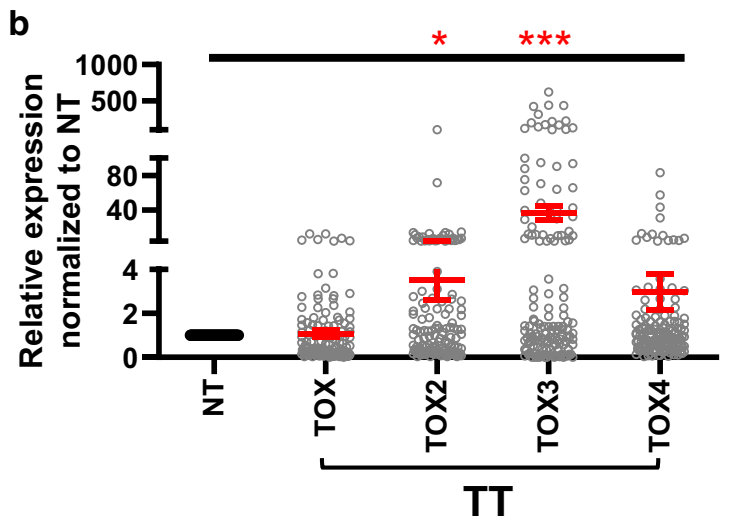

TOX2, TOX3, and TOX4) (b), and T cell exhaustion-related markers (SIRT1, Ki-67, Helios and PRDM1) (c). Scatter plots show expression levels in TT normalized to NT. The relative expression of each gene was calculated by normalization with $\beta$-actin. Each dot represents the relative expression value of a particular gene of an individual patient. Mean \pm standard error of the mean (SEM) is shown in red 


\section{Comparative analyses of ICs and T cell exhaustion markers in tumor tissues from CRC patients with early and advanced stages}

Tumor tissues are heterogeneous; different tumor types and at various pathological stages can express different levels of inhibitory ICs, transcription factors and other molecules to escape immunosurveillance and promote tumor growth [26]. Therefore, we investigated whether there were any differences in mRNA levels of different genes between early and advanced disease stages (Fig. 2). We found that PD-1 mRNA level was significantly higher in early stages, compared to advanced stages $(11.8 \pm 4.5$ vs. $3.6 \pm 6.5$, Fig. $2 a)$. Although TIM-3, CTLA-4, VISTA, and TIGIT mRNA levels were increased in advanced stages, results did not reach statistical significance (Fig. 2a). LAG-3 mRNA level showed a trend towards increased expression in early disease stages, compared to advanced stages (Fig. 2a). Similarly, CD160 mRNA level was increased in early stages, compared to advanced stages $(30.9 \pm 16.2$ vs. $1.8 \pm 0.5$, Fig. $2 a)$. CD244 did not show any differences with disease stages, while KLRG1 showed a trend towards increased expression in advanced stages (Fig. 2a). Notably, TOX mRNA level was significantly increased in advanced stages, compared to early stages $(1.4 \pm 0.33$ vs. $0.49 \pm 0.11$, Fig. $2 b)$. TOX2 showed a trend towards increased expression in advanced stages (Fig. 2b), while TOX3 and TOX4 mRNA levels did not show any differences within disease stages (Fig. 2b). Although
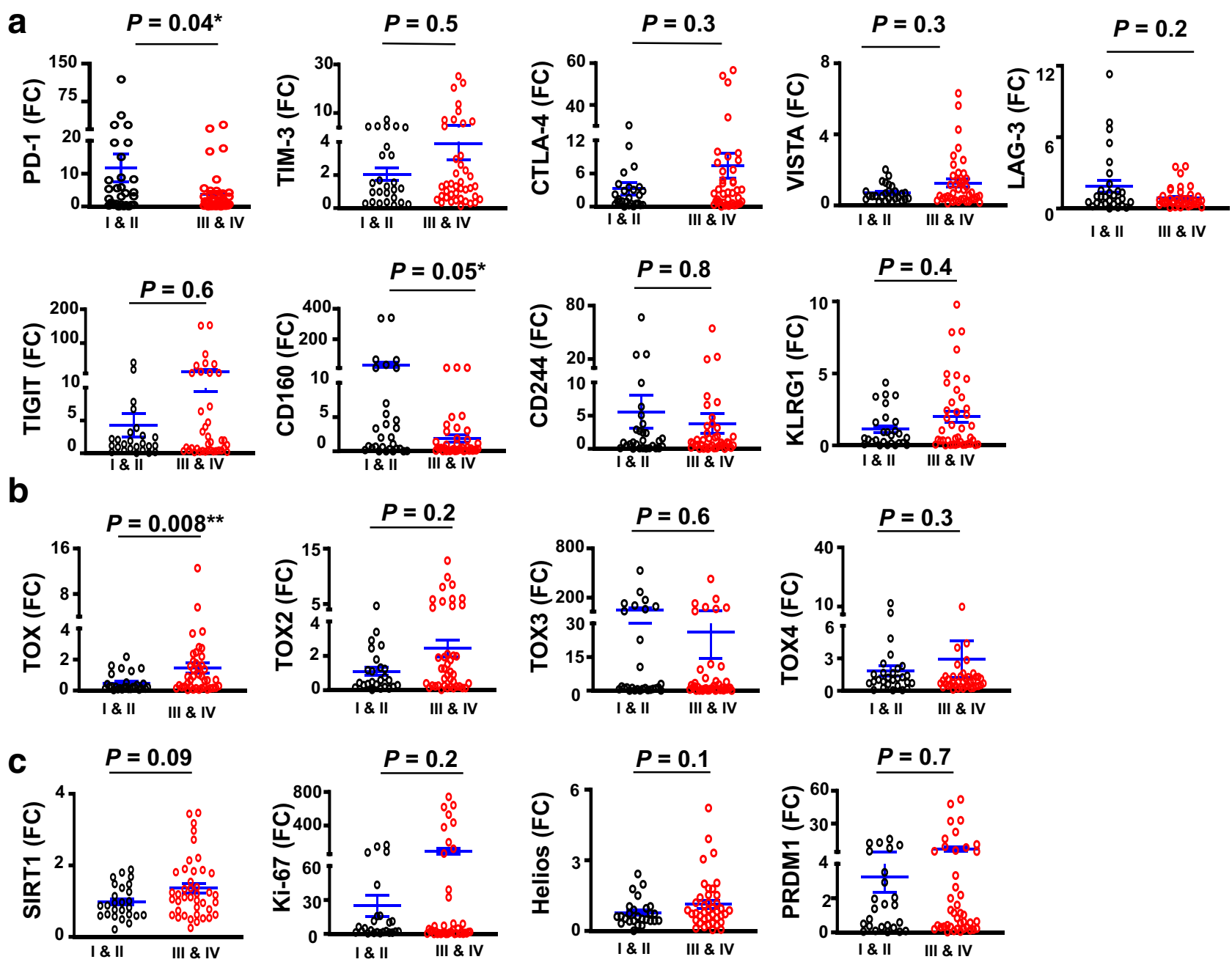

Fig. 2 Gene expression of immune checkpoints, and T cell exhaustion and cancer progression-related markers in colorectal cancer tissues from patients at early and advanced disease stages. Scatter plots show the expression of markers in early (I \& II) and advanced (III \& IV) disease stages (CRC patients $n=70$, stage I, $n=5$; stage II, $n=24$; stage III, $n=30$; stage IV, $n=11)$. Scatter plots show the expression levels of immune checkpoints (PD-1, TIM-3, CTLA-4, VISTA, LAG-
3, TIGIT, CD160, CD244 and KLRG1) (a); TOX family members (TOX, TOX2, TOX3 and TOX4) (b); other T cell exhaustion markers (SIRT1, Ki-67, Helios and PRDM1) (c) in tumor tissues of CRC patients. Results are presented as fold change (FC) of gene expression in TT versus NT. Means \pm standard error of the means (SEM) are depicted on the scatter plots 
mRNA levels of SIRT1, Ki-67, Helios, and PRDM1 were increased in advanced stages, they did not show statistical significance (Fig. 2c).

Together, these data suggest that TOX, and potentially TIM-3, CTLA-4, VISTA, TIGIT, KLRG1, TOX2, SIRT1, Ki-67, and Helios mRNA levels in CRC tumor tissues increase in advanced disease stages (Table 2), suggesting their possible roles in CRC progression. On the other hand, PD-1 and CD160 mRNA levels were higher in CRC tumor tissues from patients with early stages, indicating that targeting these two ICs in early stages could be more effective than in advanced stages.

\section{Expression of immune checkpoints and $\mathrm{T}$ cell exhaustion-related genes in the circulation of CRC patients}

Increased expression of immune checkpoints and $\mathrm{T}$ cell exhaustion markers in the circulation of colorectal cancer patients have been associated with the suppression of antitumor immunity [27]. Due to the limited RNA obtained from patient samples, we had to prioritize the genes and select a subset of genes from the panel of genes included in CRC tissue investigations, and determine their expression levels in the peripheral blood mononuclear cells (PBMCs) of CRC patients. We selected the most common ICs, some of which are being targeted in ongoing clinical trials or are approved for cancer immunotherapy, including TIM-3, LAG-3, TIGIT, VISTA, CTLA-4 and PD-1 [2]. In addition, we selected some markers that could be of interest, given their recent emerging roles in $\mathrm{T}$ cell exhaustion, such as TOX and TOX2 [14], and SIRT1, which has been implicated in CRC progression [15, 28].

We found that the mRNA expression levels of PD-1, VISTA, and LAG-3 were significantly elevated in the circulation of CRC patients, compared to healthy individuals (Fig. 3a). A trend towards an upregulated level of TOX2 was seen in the circulation of CRC patients. TIM-3 mRNA levels were relatively similar in both groups (Fig. 3a). Although CTLA-4 mRNA level was elevated in the circulation of CRC patients, it did not reach statistical significance (Fig. 3a). In contrast, TIGIT, TOX and SIRT1 mRNA levels were significantly reduced in the circulation of CRC patients (Fig. 3a). Next, we investigated differences in the expression levels of these genes between early and advanced disease stages in the circulation of CRC patients. PD-1, CTLA-4, and TIGIT expression levels were significantly higher in early stages compared with advanced stages $(2.5 \pm 0.3$ vs. $1.6 \pm 0.3$, $1.6 \pm 0.3$ vs. $0.9 \pm 0.1$, and $0.9 \pm 0.02$ vs. $0.4 \pm 0.1$, respectively, Fig. 3b). Levels of other genes including TIM-3, VISTA, TOX, TOX2 and SIRT1 were relatively similar in early and advanced stages (Fig. 3b), possibly suggesting that their expressions in the circulation have no prognostic value.

Table 2 Summary for gene expression and comparative analyses in the tumor tissue and circulation of CRC patients with early and advanced stages

\begin{tabular}{|c|c|c|c|c|}
\hline Gene & $\begin{array}{l}\text { Level in CRC TT com- } \\
\text { pared to NT }\end{array}$ & $\begin{array}{l}\text { Gene level in advanced stages } \\
\text { compared to early stages (TT) }\end{array}$ & $\begin{array}{l}\text { Level in CRC PBMC com- } \\
\text { pared to healthy donors }\end{array}$ & $\begin{array}{l}\text { Gene level in advanced stages } \\
\text { compared to early stages (circula- } \\
\text { tion) }\end{array}$ \\
\hline PD-1 & Upregulated $* * *$ & Downregulated* & Upregulated $* * *$ & Downregulated* \\
\hline TIM-3 & Upregulated $* * *$ & Upregulated (n.s) & No change & No change \\
\hline VISTA & No change & Upregulated (n.s) & Upregulated $* * *$ & No change \\
\hline CTLA-4 & Upregulated $* * *$ & Upregulated (n.s) & Upregulated (n.s) & Downregulated* \\
\hline LAG-3 & No change & Downregulated (n.s) & Upregulated $* * *$ & No change \\
\hline TIGIT & Upregulated $* * *$ & Upregulated (n.s) & Downregulated $* *$ & Downregulated $* *$ \\
\hline TOX & No change & Upregulated $* *$ & Downregulated $* * *$ & No change \\
\hline TOX2 & Upregulated* & Upregulated (n.s) & Upregulated (n.s) & No change \\
\hline TOX3 & Upregulated $* * *$ & No change & N.A & N.A \\
\hline TOX4 & Upregulated (N.S) & No change & N.A & N.A \\
\hline SIRT1 & Upregulated* & Upregulated (n.s) & Downregulated $* * *$ & No change \\
\hline Ki-67 & Upregulated $* * *$ & Upregulated (n.s) & N.A & N.A \\
\hline Helios & No change & Upregulated (n.s) & N.A & N.A \\
\hline PRDM1 & Upregulated $* *$ & Upregulated (n.s) & N.A & N.A \\
\hline KLRG1 & Upregulated* & Upregulated (n.s) & N.A & N.A \\
\hline CD160 & Upregulated* & Downregulated* & N.A & N.A \\
\hline CD244 & Upregulated* & No change & N.A & N.A \\
\hline
\end{tabular}

$C R C$ colorectal cancer, TT tumor tissue, $P B M C$ peripheral blood mononuclear cells, N.S not significant, N.A not available

$* * * P<0.001, * * P<0.01, * P \leq 0.05$ 


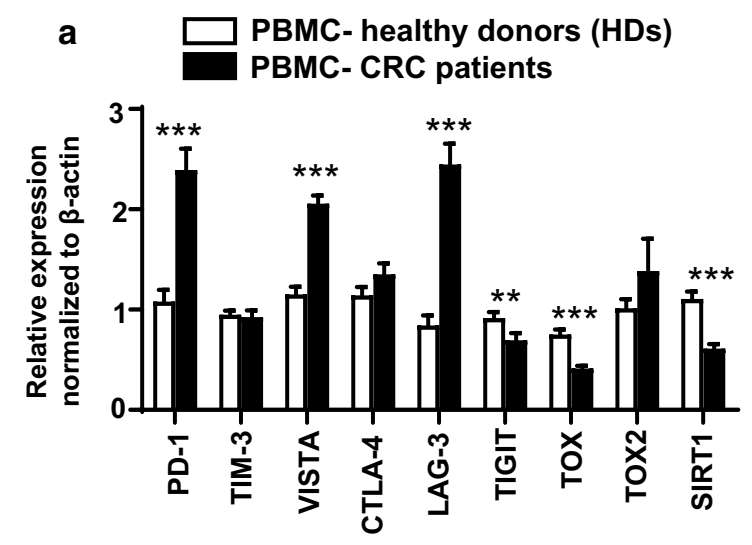

b
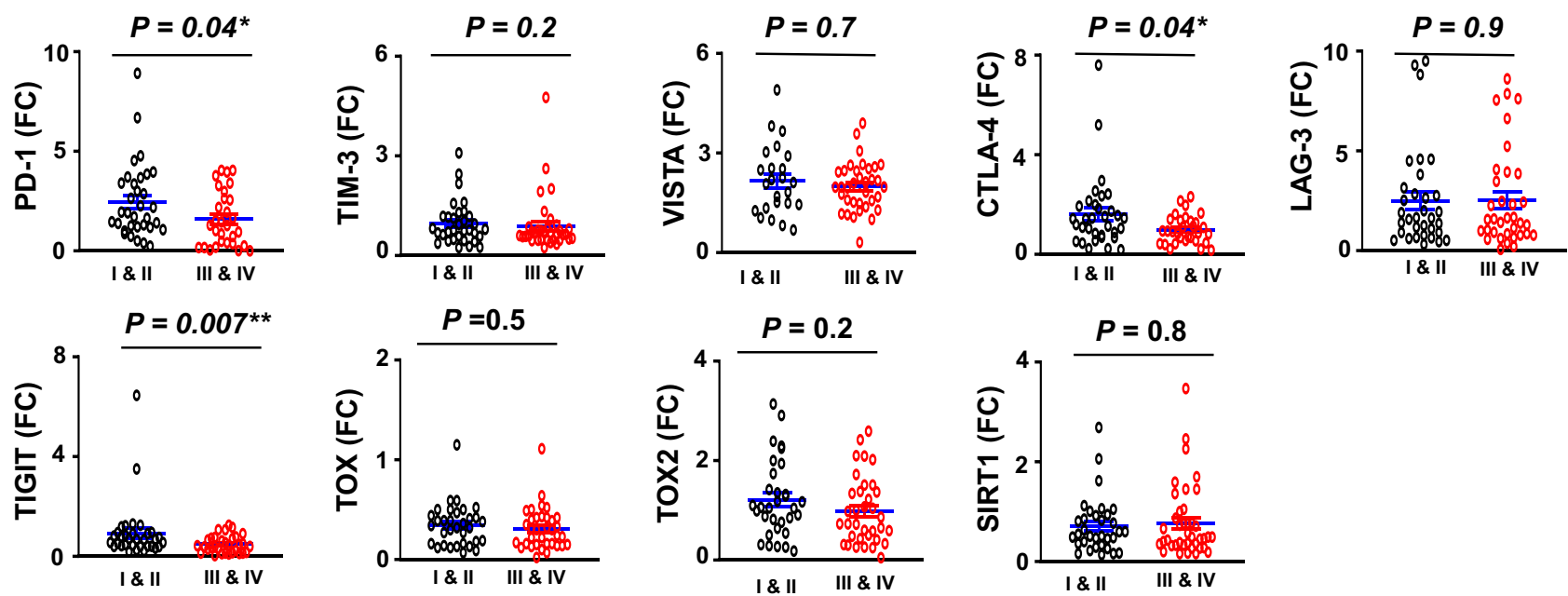

Fig. 3 Gene expression of immune checkpoints, T cell exhaustion and cancer progression-related markers in the circulation of colorectal cancer patients at early and advanced disease stages. RNA was isolated from peripheral blood mononuclear cells (PBMCs) of 30 healthy donors and $68 \mathrm{CRC}$ patients, and reverse transcribed to cDNA. Quantitative RT-PCR was performed to assess the expression levels of immune checkpoints (PD-1, TIM-3, VISTA, CTLA-4, LAG-3, and TIGIT) and T cell exhaustion markers (TOX, TOX2 and

\section{Increased expression of TIM-3, CTLA-4, TIGIT, TOX, and SIRT1 mRNA in the tumor tissue compared to the circulation of CRC patients}

Since we detected the levels of common $\mathrm{T}$ cell exhaustion markers in the tumor tissue and circulation of CRC patients, we performed a paired comparison between the circulation and tumor tissues based on the level of these genes (Fig. 4). Genes including TIM-3, CTLA-4, TIGIT, TOX, and SIRT1 showed higher levels in the TME, compared with circulation. PD-1 and TOX2 showed a trend towards an upregulated level in the TME, compared to circulation. Only two genes (VISTA and LAG-3) showed different patterns, where levels were significantly higher in the circulation, compared with the TME.
SIRT1). Bar plot shows relative expression of each gene, normalized to average expression level recorded for healthy donors (a). Scatter plots show the expression levels of PD-1, TIM-3, VISTA, CTLA-4, LAG-3, TIGIT, TOX, TOX2, and SIRT1 between early (I \& II) and advanced (III \& IV) disease stages (stage I, $n=10$; stage II, $n=23$; stage, III $n=24$; stage IV, $n=11$ ) (b). Results are presented as fold change (FC) of gene expression in CRC versus HD. Results are presented as mean \pm standard error of the mean (SEM)

Taken together, these data indicate the differential expression of $\mathrm{T}$ cell exhaustion markers in the TME and circulation of CRC patients (summarized in Table 2). This may suggest that elevated expression of PD-1, TIM-3, CTLA-4, TIGIT, TOX, TOX2, and SIRT1 genes in the TME of CRC is induced by tumor-associated, factors, unlike VISTA, and LAG-3.

\section{Comparative analyses of marker expression in the tumor tissue of CRC patients with different grades of tumor budding}

Tumor budding, evidenced by the presence of individual cells and small clusters of tumor cells at the invasive front of carcinomas, has gained attention, particularly in CRC 
Fig. 4 Paired comparison of gene expression levels in the circulation and tumor tissues of CRC patients. Scatter plots show the paired comparison between the circulation and TT of 56 CRC patients based on the expression level of PD-1, TIM-3, VISTA, CTLA-4, LAG3, TIGIT, TOX, TOX2, and SIRT1. Results are presented as fold change (FC) of gene expression in TT versus NT for tissue and CRC versus HD for circulation
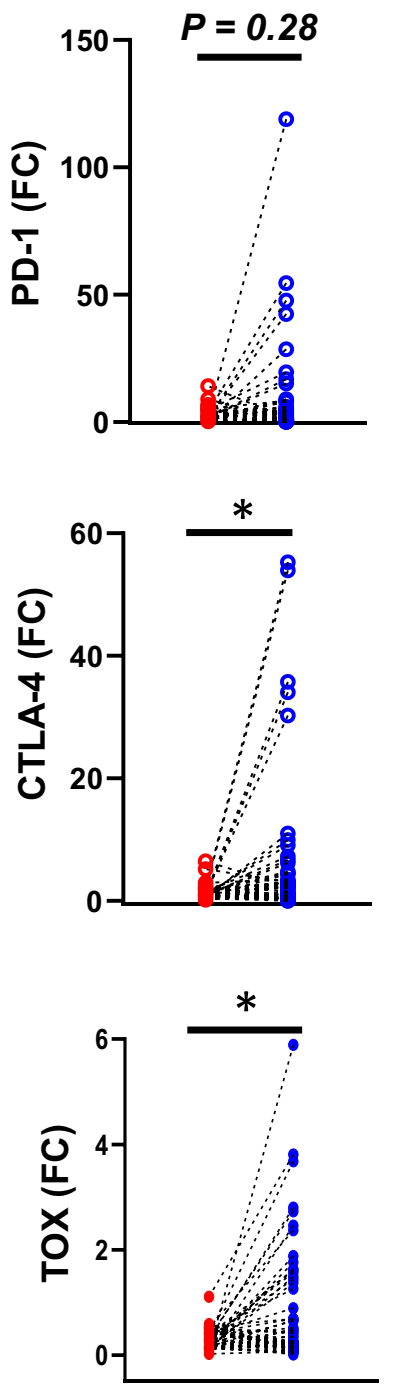

[29]. Tumor budding is an independent prognostic marker for $\mathrm{CRC}$ and important indicator of increased risk of nodal metastasis and poor disease outcomes [30]. Some clinicians and pathologists are considering that the use of tumor budding could be more helpful than using TNM staging in patient stratification into risk categories [29]. TNM staging and tumor budding represent different clinicopathological measures; they are independent of each other with different prognostic values.

Here, we investigated whether there are any differences in mRNA levels of genes investigated in TT with the grade of tumor budding, classified as low/intermediate/high (Supplementary Figure 1). PD-1, TIM-3, CTLA-4, LAG-3, and CD244 mRNA levels were relatively similar amongst the different grades of tumor budding (Supplementary
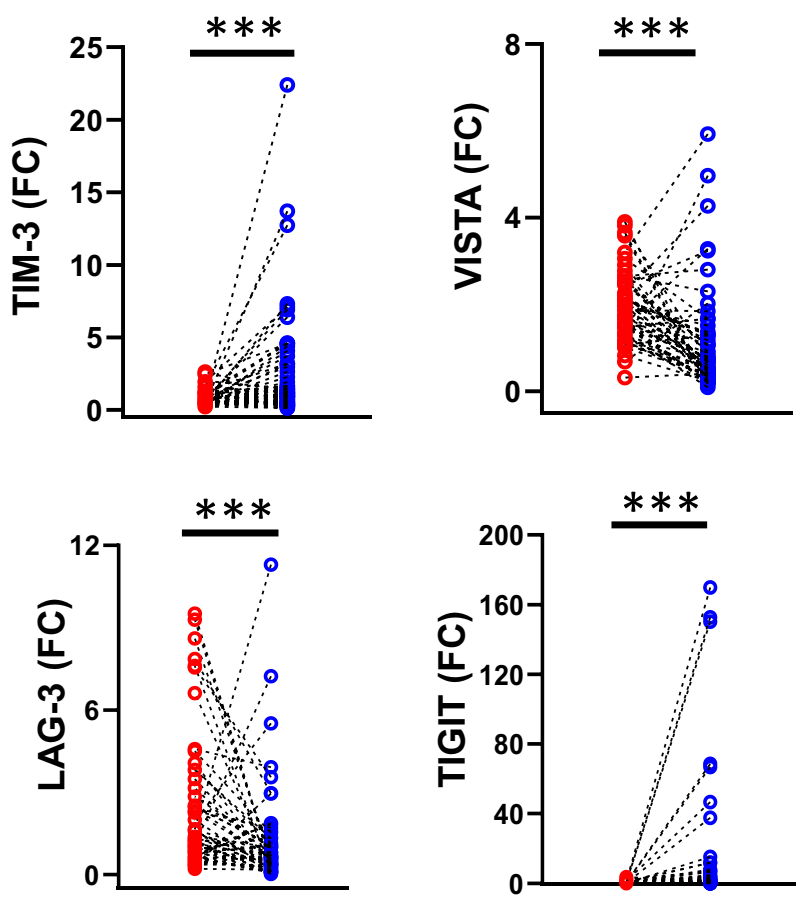

\section{- Circulation $\circ$ TT}

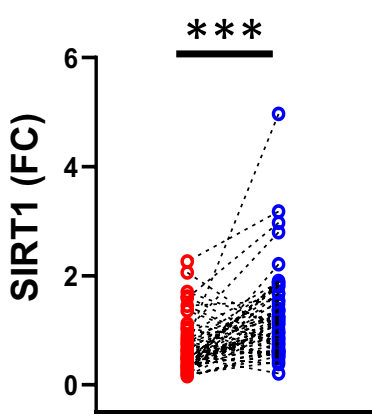

Figure 1A). VISTA, TIGIT and KLRG1 showed a trend towards increased expression in patients with high grades of tumor budding (Supplementary Figure 1A). However, level of CD160 mRNA was significantly increased in patients with low grades of tumor budding (Supplementary Figure 1A). TOX, TOX2, TOX3, and TOX4 (Supplementary Figure 1B), in addition to SIRT1, Ki-67, Helios, and PRDM1 mRNA levels (Supplementary Figure 1C) showed no differences with grades of tumor budding.

Collectively, these data suggest that mRNA levels of VISTA, TIGIT and KLRG1 in CRC tumor tissues could be elevated in CRC patients with high grades of tumor budding, which is considered as a risk factor for poor disease prognosis and metastasis in CRC patients. On the other hand, CD160 mRNA level was increased in patients with 
low grades of tumor budding. These data indicate that some IC expressions could be associated with tumor metastasis.

\section{Discussion}

Increased expression of $\mathrm{T}$ cell exhaustion markers, including ICs, in many cancer types has been previously reported [8]. We have previously reported that mRNA levels of some ICs, including PD-1, TIM-3, CTLA-4, and TIGIT, were elevated in the circulation and TME of CRC patients [21, 31]. In this study, we extended this investigation by including other $\mathrm{T}$ cell exhaustion markers, and determined their transcriptomic expressions in the TME and circulation of CRC patients at early and advanced disease stages to identify potential prognostic biomarkers and/or therapeutic targets for CRC patients. Transcriptomic changes of these markers with pathological stages and tumor budding grades in CRC patients have not been studied before.

We found that mRNA levels of PD-1, TIM-3, CTLA4, TIGIT, CD160, CD244, and KLRG1 were elevated in CRC tumor tissue, implicating their potential contribution to CRC development and/or progression. Signaling pathways of these ICs have been associated with increased suppressive activity of $\mathrm{T}$ regulatory cells (Tregs), impaired activation of antigen-presenting cells and $\mathrm{T}$ effector cells, inhibition of $\mathrm{T}$ cell proliferation and reduced cytokine production $[2,3]$. In addition, the importance of PD-1 and CTLA-4 [32-34], TIM-3 [35], CD160 [12], and CD244 [11] in promoting tumor growth and progression has been reported in preclinical models. Zhou et al., showed that targeting TIGIT, using CRISPR/Cas9 system, in mouse colon cancer models inhibited tumor growth [36]. Greenberg et al., showed that KLRG1 positively regulates tumor growth/metastasis in animal models of breast and colon cancers, and melanoma, while its blockade enhances anti-tumor immunity [37].

We also found that mRNA levels of TOX2 and TOX3 were significantly higher in CRC tumor tissues, suggesting their potential roles in CRC development and/or progression. The role of TOX proteins in T cell exhaustion has recently been highlighted in several studies [9, 13, 14, 38]. Additionally, there are no studies on the expression of TOX2 and TOX3 in CRC tumor tissues. It was demonstrated that TOX and TOX2 can cooperatively work together to induce $\mathrm{T}$ cell exhaustion by upregulating IC expression in chimeric antigen receptor (CAR) T cell model, and their targeting was shown to be beneficial in enhancing anti-tumor immune responses and reducing tumor growth [14].

SIRT1, Ki-67, and PRDM1 mRNA levels were also elevated in CRC tumor tissues; these markers could contribute to CRC by promoting tumorigenesis, and inducing $\mathrm{T}$ cell exhaustion and functional impairment via indirect mechanisms [15-17, 39]. SIRT1 is a member of the sirtuins family; acts as a negative regulator for apoptosis, IL-2 production, anti-tumor T cell responses and the tumor suppressor gene p53 [40], and positively regulates tumor growth $[15,28]$. High Ki-67 index has been associated with $\mathrm{CD}^{+} \mathrm{T}$ cell exhausted phenotype in tumors [17], suggesting that increased proliferative activity is associated with functional impairment of T cells [16]. Moreover, silencing Ki-67 gene in vitro and in vivo has been shown to induce tumor cell apoptosis [41]. Blimp-1, encoded by PRDM1 gene, has also been implicated in T cell exhaustion and associated with the induction of inhibitory IC expression (such as PD-1 and TIGIT), IL-10 production and effector T cell death [39]. In addition, overexpression of Blimp-1 in breast cancer models has been associated with tumor invasiveness and metastasis [42]. The expression of Helios in CRC tumor tissues has been linked with increased stability and suppressive activity of Tregs, which in turn positively regulate cancer progression [18]. Furthermore, the expression of Helios has been shown in exhausted $\mathrm{CD} 4^{+} \mathrm{T}$ cells [43]; however, the exact pathway(s) by which Helios induces $\mathrm{T}$ cell exhaustion is yet to be determined.

Additionally, we found that PD-1 mRNA levels in CRC tumor tissue and circulation were higher in early stages, suggesting that targeting PD-1 in CRC patients with advanced stages could be less effective. In contrast to the TME, the mRNA levels of CTLA- 4 and TIGIT in the circulation of CRC patients at early stages were higher than those at advanced stages. This could suggest that $T$ cells expressing CTLA-4 and TIGIT accumulate in the circulation of CRC patients during the onset of disease, until they are recruited to the TME as disease progresses, resulting in their accumulation within the tumor tissue.

Levels of TIM-3, CTLA-4, TIGIT, TOX, TOX2, and SIRT1 genes in tumor tissue were significantly higher than that of the circulation; their expressions within the TME could be induced by tumor-mediated immunosuppression [3, 26]. These data implicate the potential importance of their elevated expression in the TME and their potential contribution to cancer progression. In support of this, the association of CRC progression with TIM-3 [44, 45] and SIRT1 [15, 28] expression in tumor tissues have been reported.

To our best knowledge, our study is the first one showing the differences in the expression of some $\mathrm{T}$ cell exhaustion markers between early and advanced disease stages in the TME and circulation of CRC patients. Collectively, our results could offer a therapeutic benefit and help in designing more effective treatment regimens for patients with advanced stages of CRC. However, further investigations are required to validate these findings in larger cohorts of patients. Additional studies are required to elucidate the mechanisms which regulate the expression of some of these markers in the tumor tissue and circulation of CRC patients. 
Acknowledgments Open Access funding provided by the Qatar National Library. We would like to thank Ms. Eleonor Dela Cruz Belita and Mr. Mark Edison Baladad from Hamad Medical Corporation for assisting in collecting patient samples, and all patients and healthy individuals for donating their samples.

Author contributions RS performed experimental work, data analyses, and wrote the manuscript. RT assisted in manuscript writing, experimental work and data analyses. ST and VN assisted in experimental work, and revised the manuscript. KM and MAP assisted in sample collection and provided the clinicopathological data. MK and MA-D contributed to sample collection, clinical data acquisition and revising the manuscript. MAN contributed to the study design, sample collection, and revising the manuscript. EE conceived the idea, designed the study, supervised the project, analyzed and interpreted data, and wrote and revised the manuscript. All authors were involved in the final approval of the manuscript.

Funding This work was supported by a start-up Grant [VR04] for Professor Eyad Elkord from Qatar Biomedical Research Institute, Qatar Foundation.

\section{Compliance with ethical standards}

Conflict of interests The authors declare no conflicts of interest.

Ethics approval and consent to participate This study was executed under ethical approvals from Hamad Medical Corporation, Doha, Qatar (Protocol No. MRC-02-18-012) and Qatar Biomedical Research Institute, Doha, Qatar (Protocol No. 2018-018).

Availability of data and material The datasets used and/or analyzed during the current study are available from the corresponding author on reasonable request.

Open Access This article is licensed under a Creative Commons Attribution 4.0 International License, which permits use, sharing, adaptation, distribution and reproduction in any medium or format, as long as you give appropriate credit to the original author(s) and the source, provide a link to the Creative Commons licence, and indicate if changes were made. The images or other third party material in this article are included in the article's Creative Commons licence, unless indicated otherwise in a credit line to the material. If material is not included in the article's Creative Commons licence and your intended use is not permitted by statutory regulation or exceeds the permitted use, you will need to obtain permission directly from the copyright holder. To view a copy of this licence, visit http://creativecommons.org/licenses/by/4.0/.

\section{References}

1. Xia A, Zhang Y, Xu J, Yin T, Lu XJ (2019) T cell dysfunction in cancer immunity and immunotherapy. Front Immunol 10:1719. https://doi.org/10.3389/fimmu.2019.01719

2. Saleh R, Elkord E (2019) Treg-mediated acquired resistance to immune checkpoint inhibitors. Cancer Lett 457:168-179. https ://doi.org/10.1016/j.canlet.2019.05.003

3. Saleh R, Elkord E (2019) Acquired resistance to cancer immunotherapy: Role of tumor-mediated immunosuppression. Semin Cancer Biol. https://doi.org/10.1016/j.semcancer.2019.07.017
4. Fares CM, Van Allen EM, Drake CG, Allison JP, Hu-Lieskovan S (2019) Mechanisms of resistance to immune checkpoint blockade: Why does checkpoint inhibitor immunotherapy not work for all patients? Am Soc Clin Oncol Educ Book 39:147164. https://doi.org/10.1200/EDBK_240837

5. Easwaran H, Tsai HC, Baylin SB (2014) Cancer epigenetics: tumor heterogeneity, plasticity of stem-like states, and drug resistance. Mol Cell 54(5):716-727. https://doi.org/10.1016/j. molcel.2014.05.015

6. Blank CU, Haining WN, Held W, Hogan PG, Kallies A, Lugli E, Lynn RC, Philip M, Rao A, Restifo NP, Schietinger A, Schumacher TN, Schwartzberg PL, Sharpe AH, Speiser DE, Wherry EJ, Youngblood BA, Zehn D (2019) Defining 'T cell exhaustion'. Nat Rev Immunol 19(11):665-674. https://doi. org/10.1038/s41577-019-0221-9

7. Wherry EJ, Kurachi M (2015) Molecular and cellular insights into T cell exhaustion. Nat Rev Immunol 15(8):486-499. https://doi. org/10.1038/nri3862

8. Thommen DS, Schumacher TN (2018) T cell dysfunction in cancer. Cancer Cell 33(4):547-562. https://doi.org/10.1016/j.ccell .2018.03.012

9. Khan O, Giles JR, McDonald S, Manne S, Ngiow SF, Patel KP, Werner MT, Huang AC, Alexander KA, Wu JE, Attanasio J, Yan P, George SM, Bengsch B, Staupe RP, Donahue G, Xu W, Amaravadi RK, Xu X, Karakousis GC, Mitchell TC, Schuchter LM, Kaye J, Berger SL, Wherry EJ (2019) TOX transcriptionally and epigenetically programs CD8+ T cell exhaustion. Nature 571(7764):211-218. https://doi.org/10.1038/s41586-019-1325-x

10. Li L, Wan S, Tao K, Wang G, Zhao E (2016) KLRG1 restricts memory T cell antitumor immunity. Oncotarget 7(38):6167061678. https://doi.org/10.18632/oncotarget.11430

11. Agresta L, Hoebe KHN, Janssen EM (2018) The emerging role of CD244 signaling in immune cells of the tumor microenvironment. Front Immunol 9:2809. https://doi.org/10.3389/fimmu .2018.02809

12. Paulos CM, June CH (2010) Putting the brakes on BTLA in T cell-mediated cancer immunotherapy. J Clin Invest 120(1):76-80. https://doi.org/10.1172/JCI41811

13. Alfei F, Kanev K, Hofmann M, Wu M, Ghoneim HE, Roelli P, Utzschneider DT, von Hoesslin M, Cullen JG, Fan Y, Eisenberg V, Wohlleber D, Steiger K, Merkler D, Delorenzi M, Knolle PA, Cohen CJ, Thimme R, Youngblood B, Zehn D (2019) TOX reinforces the phenotype and longevity of exhausted T cells in chronic viral infection. Nature 571(7764):265-269. https://doi. org/10.1038/s41586-019-1326-9

14. Seo H, Chen J, Gonzalez-Avalos E, Samaniego-Castruita D, Das A, Wang YH, Lopez-Moyado IF, Georges RO, Zhang W, Onodera A, Wu CJ, Lu LF, Hogan PG, Bhandoola A, Rao A (2019) TOX and TOX2 transcription factors cooperate with NR4A transcription factors to impose CD8(+) T cell exhaustion. Proc Natl Acad Sci U S A 116(25):12410-12415. https://doi.org/10.1073/ pnas. 1905675116

15. Chen X, Sun K, Jiao S, Cai N, Zhao X, Zou H, Xie Y, Wang Z, Zhong M, Wei L (2014) High levels of SIRT1 expression enhance tumorigenesis and associate with a poor prognosis of colorectal carcinoma patients. Sci Rep 4:7481. https://doi.org/10.1038/srep0 7481

16. Miller BC, Sen DR, Al Abosy R, Bi K, Virkud YV, LaFleur MW, Yates KB, Lako A, Felt K, Naik GS, Manos M, Gjini E, Kuchroo JR, Ishizuka JJ, Collier JL, Griffin GK, Maleri S, Comstock DE, Weiss SA, Brown FD, Panda A, Zimmer MD, Manguso RT, Hodi FS, Rodig SJ, Sharpe AH, Haining WN (2019) Subsets of exhausted CD8(+) T cells differentially mediate tumor control and respond to checkpoint blockade. Nat Immunol 20(3):326-336. https://doi.org/10.1038/s41590-019-0312-6 
17. Song Q, Shi F, Adair M, Chang H, Guan X, Zhao Y, Li Y, Wu $\mathrm{G}, \mathrm{Wu}$ J (2019) Cell counts, rather than proportion, of CD8/PD-1 Tumor-infiltrating lymphocytes in a tumor microenvironment associated with pathological characteristics of Chinese invasive ductal breast cancer. J Immunol Res 2019:8505021. https://doi. org/10.1155/2019/8505021

18. Syed Khaja AS, Toor SM, El Salhat H, Ali BR, Elkord E (2017) Intratumoral FoxP3(+)Helios(+) regulatory T Cells upregulating immunosuppressive molecules are expanded in human colorectal cancer. Front Immunol 8:619. https://doi.org/10.3389/fimmu .2017 .00619

19. Aibar S, Abaigar M, Campos-Laborie FJ, Sanchez-Santos JM, Hernandez-Rivas JM, De Las RJ (2016) Identification of expression patterns in the progression of disease stages by integration of transcriptomic data. BMC Bioinf 17(Suppl 15):432. https://doi. org/10.1186/s12859-016-1290-4

20. Rahimi A, Gonen M (2018) Discriminating early- and late-stage cancers using multiple kernel learning on gene sets. Bioinformatics 34(13):i412-i421. https://doi.org/10.1093/bioinformatics/ bty 239

21. Elashi AA, Sasidharan Nair V, Taha RZ, Shaath H, Elkord E (2019) DNA methylation of immune checkpoints in the peripheral blood of breast and colorectal cancer patients. Oncoimmunology 8(2):e1542918. https://doi.org/10.1080/2162402X.2018.1542918

22. Xu L, Li M, Wang M, Yan D, Feng G, An G (2014) The expression of microRNA-375 in plasma and tissue is matched in human colorectal cancer. BMC Cancer 14:714. https://doi. org/10.1186/1471-2407-14-714

23. Jiang Y, Li Y, Zhu B (2015) T-cell exhaustion in the tumor microenvironment. Cell Death Dis 6:e1792. https://doi.org/10.1038/ cddis.2015.162

24. Kahan SM, Zajac AJ (2019) Immune exhaustion: past lessons and new insights from lymphocytic choriomeningitis virus. Viruses. https://doi.org/10.3390/v11020156

25. Hong JJ, Amancha PK, Rogers K, Ansari AA, Villinger F (2013) Re-evaluation of PD-1 expression by T cells as a marker for immune exhaustion during SIV infection. PLoS ONE 8(3):e60186. https://doi.org/10.1371/journal.pone.0060186

26. Shembrey C, Huntington ND, Hollande F (2019) Impact of tumor and immunological heterogeneity on the anti-cancer immune response. Cancers (Basel). https://doi.org/10.3390/cancers110 91217

27. Toor SM, Murshed K, Al-Dhaheri M, Khawar M, Abu Nada M, Elkord E (2019) Immune checkpoints in circulating and tumorinfiltrating cd $4+\mathrm{t}$ cell subsets in colorectal cancer patients. Frontiers Immunol 10:2936

28. Yu DF, Jiang SJ, Pan ZP, Cheng WD, Zhang WJ, Yao XK, Li YC, Lun YZ (2016) Expression and clinical significance of Sirt1 in colorectal cancer. Oncol Lett 11(2):1167-1172. https://doi. org/10.3892/ol.2015.3982

29. Mitrovic B, Schaeffer DF, Riddell RH, Kirsch R (2012) Tumor budding in colorectal carcinoma: time to take notice. Mod Pathol 25(10):1315-1325. https://doi.org/10.1038/modpathol.2012.94

30. Blank A, Schenker C, Dawson H, Beldi G, Zlobec I, Lugli A (2019) Evaluation of tumor budding in primary colorectal cancer and corresponding liver metastases based on H\&E and pancytokeratin staining. Front Med (Lausanne) 6:247. https://doi. org/10.3389/fmed.2019.00247

31. Sasidharan Nair V, Toor SM, Taha RZ, Shaath H, Elkord E (2018) DNA methylation and repressive histones in the promoters of PD-1, CTLA-4, TIM-3, LAG-3, TIGIT, PD-L1, and galectin-9 genes in human colorectal cancer. Clin Epigenet 10(1):104. https ://doi.org/10.1186/s13148-018-0539-3

32. Kleffel S, Posch C, Barthel SR, Mueller H, Schlapbach C, Guenova E, Elco CP, Lee N, Juneja VR, Zhan Q, Lian CG, Thomi R, Hoetzenecker W, Cozzio A, Dummer R, Mihm MC Jr, Flaherty
KT, Frank MH, Murphy GF, Sharpe AH, Kupper TS, Schatton T (2015) Melanoma cell-intrinsic PD-1 receptor functions promote tumor growth. Cell 162(6):1242-1256. https://doi.org/10.1016/j. cell.2015.08.052

33. Grosso JF, Jure-Kunkel MN (2013) CTLA-4 blockade in tumor models: an overview of preclinical and translational research. Cancer Immun 13:5

34. Rotte A (2019) Combination of CTLA-4 and PD-1 blockers for treatment of cancer. J Exp Clin Cancer Res 38(1):255. https://doi. org/10.1186/s13046-019-1259-Z

35. Kang CW, Dutta A, Chang LY, Mahalingam J, Lin YC, Chiang JM, Hsu CY, Huang CT, Su WT, Chu YY, Lin CY (2015) Apoptosis of tumor infiltrating effector TIM-3+CD8+ T cells in colon cancer. Sci Rep 5:15659. https://doi.org/10.1038/srep15659

36. Zhou XM, Li WQ, Wu YH, Han L, Cao XG, Yang XM, Wang HF, Zhao WS, Zhai WJ, Qi YM, Gao YF (2018) Intrinsic expression of immune checkpoint molecule TIGIT could help tumor growth in vivo by suppressing the function of NK and CD8(+) T cells. Front Immunol 9:2821. https://doi.org/10.3389/fimmu .2018 .02821

37. Greenberg SA, Kong SW, Thompson E, Gulla SV (2019) Coinhibitory $\mathrm{T}$ cell receptor KLRG1: human cancer expression and efficacy of neutralization in murine cancer models. Oncotarget 10(14):1399-1406. https://doi.org/10.18632/oncotarget.26659

38. Yao C, Sun HW, Lacey NE, Ji Y, Moseman EA, Shih HY, Heuston EF, Kirby M, Anderson S, Cheng J, Khan O, Handon R, Reilley J, Fioravanti J, Hu J, Gossa S, Wherry EJ, Gattinoni L, McGavern DB, O'Shea JJ, Schwartzberg PL, Wu T (2019) Single-cell RNAseq reveals TOX as a key regulator of CD8(+) T cell persistence in chronic infection. Nat Immunol 20(7):890-901. https://doi. org/10.1038/s41590-019-0403-4

39. Zhu L, Kong Y, Zhang J, Claxton DF, Ehmann WC, Rybka WB, Palmisiano ND, Wang M, Jia B, Bayerl M, Schell TD, Hohl RJ, Zeng H, Zheng H (2017) Blimp-1 impairs T cell function via upregulation of TIGIT and PD-1 in patients with acute myeloid leukemia. J Hematol Oncol 10(1):124. https://doi.org/10.1186/ s13045-017-0486-z

40. Warren JL, MacIver NJ (2019) Regulation of adaptive immune cells by sirtuins. Front Endocrinol (Lausanne) 10:466. https://doi. org/10.3389/fendo.2019.00466

41. Li LT, Jiang G, Chen Q, Zheng JN (2015) Ki67 is a promising molecular target in the diagnosis of cancer (review). Mol Med Rep 11(3):1566-1572. https://doi.org/10.3892/mmr.2014.2914

42. Sciortino M, Camacho-Leal MdP, Orso F, Grassi E, Costamagna A, Provero P, Tam W, Turco E, Defilippi P, Taverna D, Cabodi S (2017) Dysregulation of Blimp1 transcriptional repressor unleashes p130Cas/ErbB2 breast cancer invasion. Sci Rep 7(1):1145. https://doi.org/10.1038/s41598-017-01332-z

43. Crawford A, Angelosanto JM, Kao C, Doering TA, Odorizzi PM, Barnett BE, Wherry EJ (2014) Molecular and transcriptional basis of CD4(+) T cell dysfunction during chronic infection. Immunity 40(2):289-302. https://doi.org/10.1016/j.immuni.2014.01.005

44. Xu B, Yuan L, Gao Q, Yuan P, Zhao P, Yuan H, Fan H, Li T, Qin P, Han L, Fang W, Suo Z (2015) Circulating and tumor-infiltrating Tim-3 in patients with colorectal cancer. Oncotarget 6(24):2059220603. https://doi.org/10.18632/oncotarget.4112

45. Zhou E, Huang Q, Wang J, Fang C, Yang L, Zhu M, Chen J, Chen L, Dong M (2015) Up-regulation of Tim-3 is associated with poor prognosis of patients with colon cancer. Int J Clin Exp Pathol 8(7):8018-8027

Publisher's Note Springer Nature remains neutral with regard to jurisdictional claims in published maps and institutional affiliations. 